\title{
Sind wir noch zu retten?
}

\section{Probleme des frühzeitigen Ausstiegs aus dem Pflegeberuf Ist der frühzeitige Ausstieg} aus dem Pflegeberuf eine Bedrohung für den Berufsstand? Was kann getan werden, um den Pflegeberuf und somit auch die pflegerische Versorgung von Patienten und Pflegebedürftigen zukünftig sicherzustellen? Was müssen Arbeitgeber tun, um qualifizierte Pflegekräfte an sich zu binden? Diesen Fragen ist die Autorin in einer Literaturarbeit im Rahmen ihrer Fachweiterbildung für Anästhesie- und Intensivpflege nachgegangen und hat damit beim Thieme Intensiv-Pflegepreis 2016 den 1. Platz belegt. Die Kernfrage Ihres Beitrags lautet: Wer pflegt uns 2050? 
- Nina Rammler -

Aufgrund der Alterung der Gesellschaft wird sich der Gesundheits- und Pflegeversorgungsbedarf weiter erhöhen, während sich der Pflegenachwuchs zahlenmäßig verringert. Es ist davon auszugehen, dass sich der Personalmangel in der Pflege in den nächsten Jahren über die bisherigen zyklischen Verknappungen hinaus verschärft. ${ }^{1}$

\section{Drohender Mangel an \\ Pflegefachkräften \\ Demografischer Wandel}

Menschen, die in den geburtenstarken Jahrgängen der 1960er-Jahre auf die Welt kamen, sind heute 50 Jahre alt. Ihr Anteil ist in den Bevölkerungspyramiden deutlich erkennbar ( $\rightarrow$ Abb. 1). Diese geburtenstarken Jahrgänge werden voraussichtlich um 2030 aus dem Berufsleben ausscheiden. Seit dem Ende der Sechzigerjahre ist ein stetiger Geburtenrückgang zu verzeichnen. ${ }^{2}$ Diese rückläufige Geburtenrate steht einer höheren Lebenserwartung gegenüber. Im Vergleich zu 2009 wird Berechnungen zufolge die Zahl der Pflegebedürftigen bis zum Jahr 2050 um etwa 50\% auf 4,5 Millionen ansteigen. Dies hat direkte Auswirkungen auf den Bedarf an qualifiziertem Pflegepersonal, der somit im Laufe der Jahre kontinuierlich ansteigen wird.

Der demografische Wandel bedeutet aber nicht nur, dass die Bevölkerung insgesamt älter wird, auch das Alter der Pflegekräfte steigt an. Zugleich wird die Zahl derer, die im Pflegeberuf arbeiten, abnehmen, sodass es im Jahr 2030 bis zu 500.000 offene Arbeitsstellen im Bereich der Pflege geben könnte. ${ }^{3}$ Durch den Stellenabbau im Pflegebereich in den Jahren 1996-2008 wird dieses Phänomen zusätzlich verstärkt. Der Altersdurchschnitt im Pflegeberuf erhöht sich dadurch schneller als in der Gesamtbevölkerung. ${ }^{4}$

\section{Fachkräftemangel}

Bereits jetzt kann von einem Mangel an examinierten Fachpflegekräften ausgegangen werden. Arbeitgeber signalisieren, dass es zunehmend schwerfällt, offene Stellen zu besetzen. Die Engpassanalyse der Bundesagentur für Arbeit liefert die wohl genauesten Zahlen. Basis hierfür sind gemeldete offene Arbeitsstellen und regis-

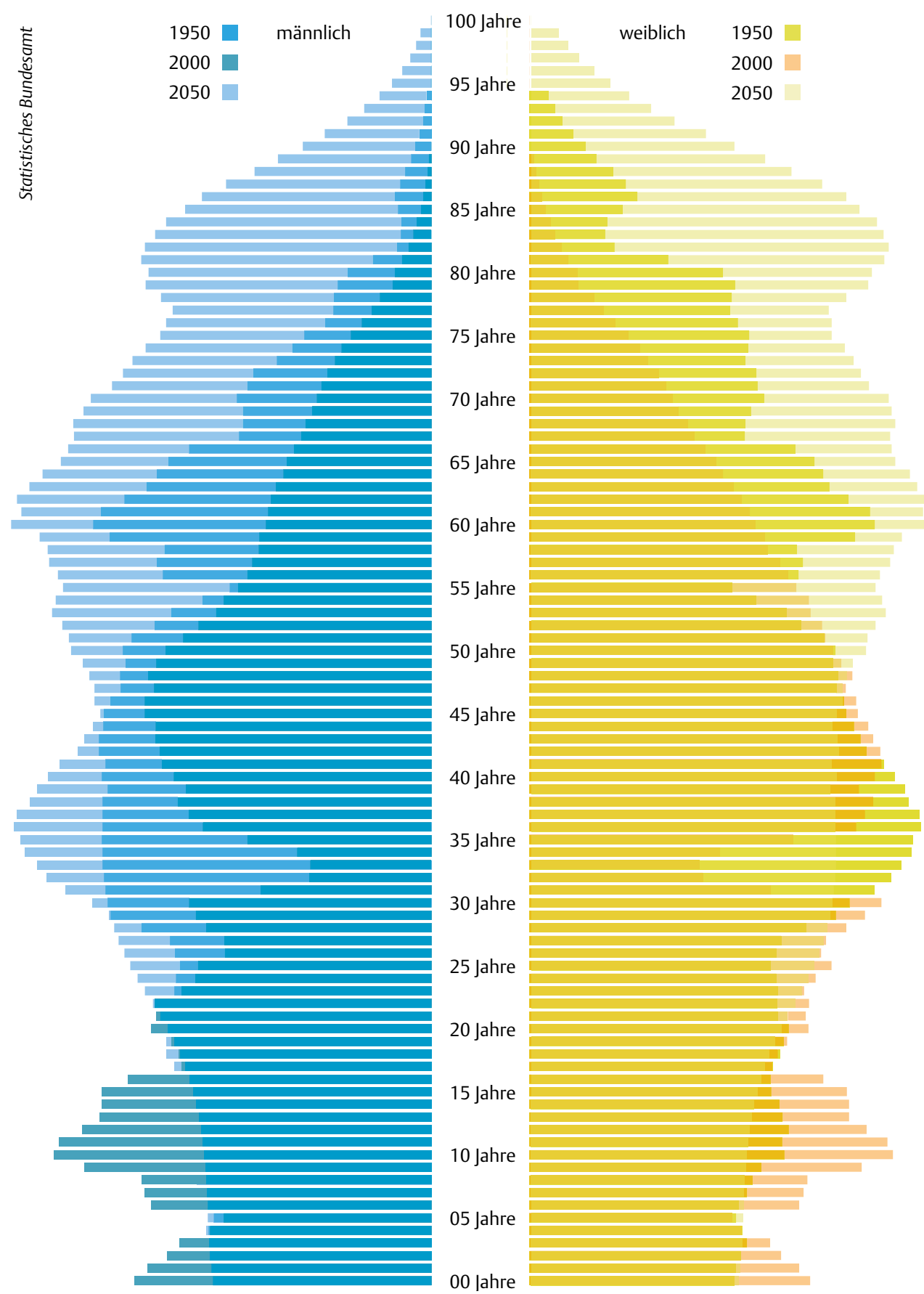

Abb. 1 Demografische Entwicklung in Deutschland 1950, 2000 und 2050 - eigene Darstellung.

trierte Arbeitslose. Allerdings kann hier von einer Abweichung zur realen Situation ausgegangen werden, da es in Deutschland keine Meldepflicht für offene Arbeitsstellen gibt. Schätzungen der Bundesagentur für Arbeit zufolge wird lediglich die Hälfte der offenen Stellen gemeldet.5,6

Kamen 2014 auf 100 gemeldete offene Stellen in der Gesundheits- und Kran- kenpflege noch 82 registrierte Arbeitslose, waren es 2016 nur noch 64 Arbeitslose. Auch die Vakanzzeit (2014: 111 Tage; 2016: 132 Tage) der als offen gemeldeten Stellen im Bundesdurchschnitt macht den fortschreitenden Fachkräftemangel deutlich. Es wird zukünftig immer länger dauern, eine freie Stelle entsprechend neu zu besetzen. ${ }^{5,6}$ 
Nachwuchsmangel ein Imageproblem?

Zukünftig werden sich immer weniger Schulabgänger für eine Ausbildung in einem Pflegeberuf entscheiden. Dies wird vor allem auf die stetig schlechter werdenden Arbeitsbedingungen in der Pflege zurückgeführt. ${ }^{7}$

Der Pflegeberuf gehört nicht zu den favorisierten Berufen der zukünftigen Schulabgänger. Nur knapp 7\% ziehen ernsthaft die Wahl eines Pflegeberufs in Betracht. Hierbei sind es eher die Absolventen der Mittelschulen (11,4\%), gefolgt von Realschülern (5,3\%) und Gymnasiasten $(3,4 \%)$. Ebenso zeigt sich, dass Faktoren wie Einkommen, Arbeitsplatzsicherheit, Arbeitsqualität und Aufstiegsmöglichkeiten bei der Berufswahl von Bedeutung sind. ${ }^{8}$

\section{Gründe für einen frühzeitigen \\ Ausstieg aus dem Pflegeberuf}

In der als Längsschnittstudie angelegten Nurse's Early Exit Study (NEXT-Studie) wurden die Ursachen, Umstände und Folgen des vorzeitigen Berufsausstiegs aus dem Pflegeberuf untersucht. In zehn europäischen Ländern wurden zeitgleich 78.000 anonymisierte Fragebögen an Pflegekräfte versendet und von annähernd 40.000 Pflegenden beantwortet.

Die Befragung enthielt Fragen zum Arbeits- und Privatleben und $\mathrm{zu}$ den $\mathrm{Zu}-$ kunftsperspektiven der Teilnehmer. Teilnehmende Pflegende, die ihre Einrichtung innerhalb der darauffolgenden zwölf Monate verlassen haben, erhielten weitere Fragebögen, um Gründe für ihr Ausscheiden und die Folgen und Konsequenzen ihres Ausstiegs zu untersuchen.

Pflegende, die in ihren Einrichtungen geblieben sind, erhielten zwölf Monate nach der Befragung einen abschließenden Fragebogen, der sich mit möglichen Veränderungen der relevanten Umstände und deren Auswirkung auf eine dauerhafte Ausübung des Pflegeberufs befasste. ${ }^{9}$

Faktoren, die zu einem frühzeitigen Berufsausstieg beitragen können, sind:

- Gesundheitliche Gründe und körperliche Belastungen

- Familiäre und soziale Gründe

- Weiterqualifikation und Weiterbildung

- Betriebliche Gründe
Diese einzelnen Faktoren können nicht isoliert betrachtet werden, sondern stehen immer miteinander in Verbindung.

Ein wichtiger Faktor bezüglich der $\mathrm{Ar}$ beitszufriedenheit und Gesundheit ist die Arbeitszeitgestaltung. Diese beinhaltet Arbeitsstunden, Schichtdienst, Regelmäßigkeit und Vorhersehbarkeit von Dienstzeiten und den eigenen Einfluss auf die Dienstplangestaltung.

\section{Gesundheitliche Gründe und körperliche Belastung}

Nacht- und Schichtarbeit können schädlich für die Gesundheit werden. Zu typischen Gefährdungen zählen in erster Linie Schlafstörungen bzw. gestörte Schlafverhältnisse, gastrointestinale Probleme, kardiovaskuläre Störungen sowie psychische, psychosomatische und onkologische Erkrankungen. Frauen haben häufig Menstruationsbeschwerden, zudem steigt das Risiko von Fehlgeburten. ${ }^{9}$

Dienstpläne bestimmen die Freizeitgestaltung der Beschäftigten. Die besonderen Arbeitszeiten haben Auswirkungen auf den gesamten Alltag der Schichtarbeiter und können neben den gesundheitlichen auch zu sozialen Problemen führen. ${ }^{9}$

Mitarbeiter des Pflegedienstes müssen zu jeder Tages- und Nachtzeit verfügbar sein, um die medizinisch-pflegerische Versorgung der Patienten je nach Bedarf sicherzustellen. Schicht-, aber auch Bereitschafts- und Rufbereitschaftsdienste sind gängige Modelle, um beispielsweise Spitzenzeiten oder Notfallsituationen abzudecken. ${ }^{10}$ Dies beeinträchtigt die Lebensqualität der Beschäftigten sowie deren Familien zum Teil erheblich. Eine optimale Gestaltung der Nacht- und Schichtarbeit sollte sowohl den Gesundheitsschutz der Beschäftigten als auch die betrieblichen Erfordernisse sicherstellen. ${ }^{11}$

Schichtarbeit kann sowohl die Gesundheit der Mitarbeiter beeinträchtigen als auch zu einem Konflikt zwischen Arbeit und Familie führen und somit gleich mehrere Faktoren für einen potenziellen Berufsausstieg betreffen.

Auch die körperliche Belastung kann eine Auswirkung auf die Arbeitszufriedenheit haben. Über $80 \%$ der befragten deutschen Pflegekräfte gaben an, mit der bestehenden körperlichen Belastung un- zufrieden zu sein. In diesem Zuge werden Tätigkeiten wie Heben, Lagern, Umsetzen und Mobilisieren von Patienten genannt. $56 \%$ der Pflegekräfte in Deutschland nehmen häufiger als sechsmal täglich eine unbequeme Körperhaltung ein. Auch das Stehen für sechs Stunden oder länger wurde hier mit $65 \%$ sehr häufig genannt. ${ }^{9}$ Pflegekräfte haben im Vergleich zur allgemeinen Bevölkerung ein 1,6-fach erhöhtes Risiko einen Bandscheibenvorfall zu erleiden. ${ }^{12}$

Datenauswertungen unterschiedlicher Krankenkassen und Rentenversicherungsträger zeigen auf, dass bei der Gruppe der Erwerbstätigen über 50 Jahre die Zahl an psychischen und physischen Erkrankungen, die zu temporärer Arbeitsunfähigkeit oder gar zu Frühberentungen führen, etwa doppelt so hoch ist wie die der restlichen sozialversicherungspflichtigen Bürger. Viele beruflich Pflegende verlassen vor dem Erreichen des Rentenalters den Pflegeberuf. Von den im Zuge der Pflegethermometererhebung befragten Pflegekräfte gaben $25 \%$ an, eine Reduzierung ihrer $\mathrm{Ar}$ beitszeit aufgrund von Überforderung anzustreben. Zusätzlich geht nur jede zweite Pflegekraft davon aus, ihren Beruf bis ins Rentenalter ausüben zu können. ${ }^{4}$

In Deutschland geben $40 \%$ der Pflegekräfte Probleme mit dem Bewegungsapparat an - im europaweiten Vergleich ist das die höchste Zahl. Diejenigen Pflegenden, die von starker körperlicher Belastung und gesundheitlichen Einschränkungen berichten, waren auch diejenigen, die einen Ausstieg aus dem Pflegeberuf in Betracht ziehen (56,7\%). ${ }^{9}$

\section{Familiäre und soziale Gründe}

Nacht- und Wochenendarbeit sind vor allem das Sozial- und Familienleben beeinflussende Stressoren. 16\% der befragten Pflegenden in Deutschland mussten mindestens dreimal pro Monat kurzfristig eine Schicht übernehmen. Dies ist im Vergleich mit anderen Ländern ein sehr hoher Anteil. Die Notwendigkeit des ständigen Abrufbarseins bedeutet einen weiteren Stressfaktor. Im Zuge der NEXT-Studie ließ sich auch ein Zusammenhang mit der Dienstplanzufriedenheit und dem Wunsch, den Beruf zu verlassen, feststellen. Von denjenigen Befragten, die mit ihren Dienstplänen nicht zufrieden waren, dachten $21 \%$ daran, den 
Pflegeberuf aufzugeben. Damit waren dies doppelt so viele wie diejenigen, die ihre Arbeitszeitgestaltung hinsichtlich ihres Privatlebens als befriedigend empfanden. ${ }^{9}$

\section{Weiterqualifikation \\ und Weiterbildung}

Das Erwägen eines vorzeitigen Berufsausstiegs bedeutet aber nicht, dass nicht auch nach Alternativen innerhalb des Pflegeberufs gesucht wird. So erwägt ein Drittel der Ausstiegswilligen die Möglichkeit der Weiterqualifizierung innerhalb ihres Berufs. Das Anbieten von Weiterbildungsmöglichkeiten gilt als gute Option, einige der ansonsten Ausstiegswilligen im Beruf zu halten. Attraktiv wird eine Weiterqualifizierung aber erst, wenn diese mit erhöhter Verantwortlichkeit bei der Arbeit und höherem Einkommen verbunden ist. ${ }^{9}$

Mehr als die Hälfte der im Pflegethermometer 2009 Befragten schätzen die Pflege als einen Beruf, in dem es gute Entwicklungschancen gibt. Der Pflegeberuf wird größtenteils nicht mehr als Sackgassenberuf angesehen. Erklärt werden kann dies neben der hohen Arbeitsplatzsicherheit durch die mannigfaltigen Handlungsfelder und Schwerpunkte, die Fort- und Weiterbildungsmöglichkeiten und die langsam zunehmenden Studienangebote. 23,7\% der Befragten können sich ein Studium im Pflegebereich vorstellen. Dies betrifft nicht nur die Berufsanfänger, sondern zieht sich durch alle berufstätigen Altersgruppen. Der Trend zum lebenslangen Lernen zeichnet sich also ab. Im Gegensatz dazu zeigt sich eine deutliche Unzufriedenheit bezüglich der Bezahlung. 86\% der befragten Pflegekräfte in Deutschland empfinden diese als nicht angemessen. ${ }^{4}$

\section{Betriebliche Gründe}

Es gibt auch Unterschiede innerhalb einzelner Institutionen. Attraktive Einrichtungen scheinen ihr Personal deutlich länger zu binden und es im Beruf zu halten. In dem Krankenhaus, in dem die meisten Pflegenden angaben, den Beruf verlassen zu wollen, betrug die Zahl derer, die in dieser Einrichtung mehr als fünf Jahre gearbeitet hatten, 44,7\%. Im Gegensatz dazu dachten Pflegende in Kliniken, in denen bis zu 82\% der Pflegekräfte länger als fünf Jahre beschäftigt sind, am seltensten an einen Berufsausstieg. ${ }^{9}$

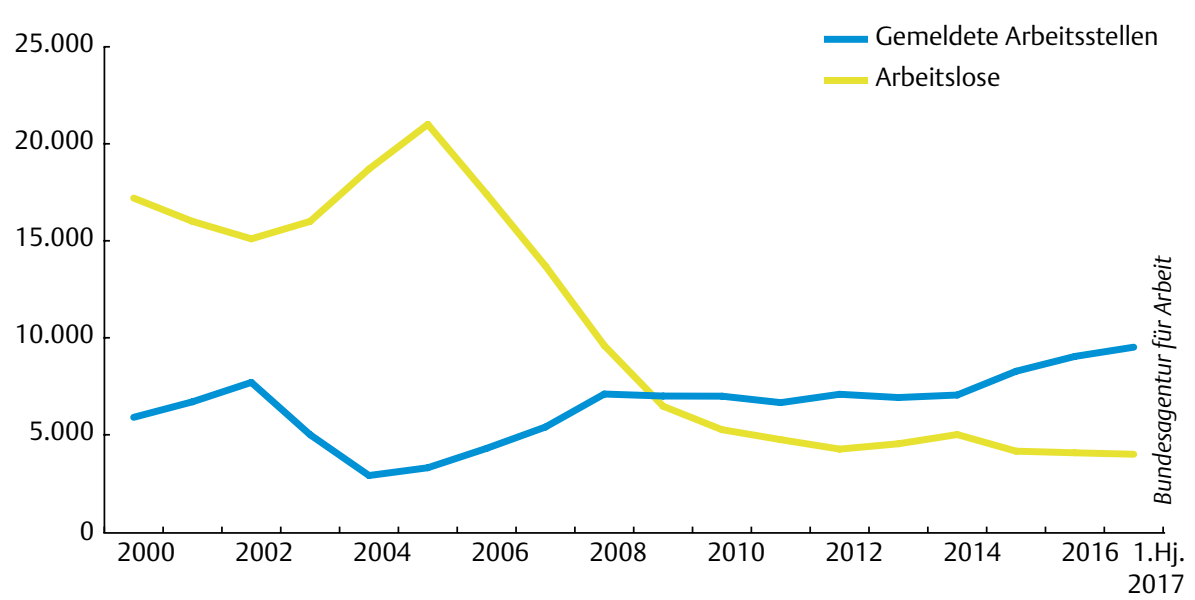

Abb. 2 Durchschnittliche Entwicklung der Arbeitsmarktsituation in der Gesundheits- und Krankenpflege - eigene Darstellung.

Wegen Personalknappheit, erhöhter Arbeitsintensität und Ausfallzeiten durch erkrankte Kollegen kommt es mehrfach zum kurzfristigen Einspringen vakanter Pflegender. Davon abgesehen, dass diese „eingesprungenen“ Tage zumeist schon privat anderweitig verplant wurden, können sie nun nicht mehr zur benötigten Erholung genutzt werden. Nur 5,6\% der Befragten gaben an, in den letzten sechs Monaten vor der Befragung keine Überstunden geleistet zu haben. Daher kann davon ausgegangen werden, dass das Erbringen von Überstunden keinesfalls eine Ausnahme, sondern eher die Regel ist. Bei nur einem Drittel der Pflegekräfte waren es unter 25 Überstunden, bei $40 \%$ häuften sich in sechs Monaten 46-70 Überstunden an. Durch die zunehmende Arbeitsbelastung können die durch diese zusätzlichen Dienste oder durch eine Verlängerung der Arbeitszeit aufgebauten Überstunden nicht zeitnah wieder abgebaut werden. Für eine unzureichende Stellenbesetzung spricht, dass dies bei den Befragten mit 58,8\% mehrheitlich der Fall ist. Auf alle Pflegenden in Deutschland hochgerechnet werden Überstunden geleistet, die dem Volumen der Stellenkürzungen in den letzten Jahren entsprechen. ${ }^{4}$

\section{Personalgewinnung}

und Personalerhaltung

Der Arbeitsmarkt im Wandel

zum Arbeitnehmermarkt

Der Arbeitsmarkt in der Pflege erlebt einen Wandel zum Arbeitnehmermarkt.
Gerade in Metropolregionen, wo es eine Vielzahl verschiedener Kliniken sowie unterschiedlicher Träger gibt, haben Bewerber freie Auswahl an geeigneten Stellen. Das spiegeln auch die statistischen Erhebungen der Bundesagentur für Arbeit wider. Die Anzahl der gemeldeten Arbeitsstellen übersteigt die der arbeitslos gemeldeten Gesundheits- und Krankenpfleger zunehmend ( $\rightarrow$ Abb. 2) ${ }^{13}$

Mehr denn je sind Arbeitgeber bei der Suche nach potenziellen Mitarbeitern in der Pflicht, die Bewerber auf sich aufmerksam zu machen und sich zudem mit Besonderheiten von der Konkurrenz abzugrenzen, um neue Mitarbeiter zu gewinnen. ${ }^{14}$

Es werden zukünftig nicht mehr die Arbeitnehmer sein, die sich bei ihrer Bewerbung um eine Stelle besonders hervorheben, sondern die Arbeitgeber, die bei den Bewerbern punkten müssen. Ebenso werden Arbeitnehmer zukünftig eher Forderungen nach besseren Arbeitsbedingungen stellen, als sie dies in der Vergangenheit getan haben. Die Veränderungen des demografischen Wandels werden dazu führen, dass qualifizierte Bewerber auf dem Arbeitsmarkt zukünftig ein knappes Gut sein werden. ${ }^{15}$

Ein weiteres Problem im Generationenwechsel ist, dass Arbeitgeber und potenzielle Arbeitnehmer scheinbar nicht mehr zusammenfinden. Eine von StepStone und dem Thieme-Verlag durchgeführte Umfrage unter in Gesundheitsberufen Beschäftigten ergab, dass das Internet die am meisten genutzte Quelle für die Suche 
nach Arbeitsplätzen ist. 60\% der Stellenangebote werden laut StepStone aber in Printmedien geschaltet. ${ }^{16}$

Printanzeigen als externes Personalmarketinginstrument in Fachzeitschriften verlieren daher aufgrund der zunehmenden Digitalisierung an Bedeutung. Betrachtet man lediglich die quantitative Qualität der Personalakquise, gehen die meisten Bewerbungen über Online-Kanäle ein. ${ }^{14}$

Ein weiterer Vorteil von Onlinestellenbörsen ist die Möglichkeit überregional auf offene Stellen im Unternehmen aufmerksam zu machen und neue Mitarbeiter zu werben. 39\% der befragten Pflegekräfte in der StepStone-Umfrage haben angegeben, dass sie für eine neue Stelle umziehen würden. ${ }^{16}$

Ein lmage ist das, was
man bräuclute, dass die anderen denken, dass man so ist, wie man gerne wäre. 17

\section{Arbeitgeber müssen überzeugen}

Schlechte Arbeitsbedingungen, Überstunden und Kommunikationsschwierigkeiten in Kliniken sprechen sich nicht nur in Internet und sozialen Netzwerken schnell herum. Auch Krankenpflegeschüler, Praktikanten und Bewerber, die sich die Klinik und deren Stationen bei Vorstellungsgesprächen, Bewerbertagen oder im Rahmen von „Probe-Arbeitstagen“ ansehen, bemerken schnell potenziell schlechtes $\mathrm{Ar}$ beitsklima und negative Stimmung. Arbeitgeber, die beliebt sind und bei denen die Mitarbeiter gern arbeiten, gelten als Marken. Es ist jedoch deutlich schwieriger für ein Unternehmen eine solch positive Stimmung in der Bewerberwelt zu positionieren.

Dieses Employer Branding lässt sich vom Unternehmen aber zumindest zum Teil steuern. Eine mehrseitige Hochglanzbroschüre mit „Vorzeige-Mitarbeiterorientierung“, in denen dem Bewerber alle erdenklichen Kriterien angepriesen werden, reicht dabei bei Weitem nicht aus, um sich auf dem Arbeitgebermarkt gegenüber der Konkurrenz behaupten zu können. Es ist unabdingbar, in Führungskräfte zu investieren. Eine Vertrauensbasis zum Vor-
Abb. 3 Modulare

Anpassung des

5-Säulen-Konzepts. ${ }^{19}$

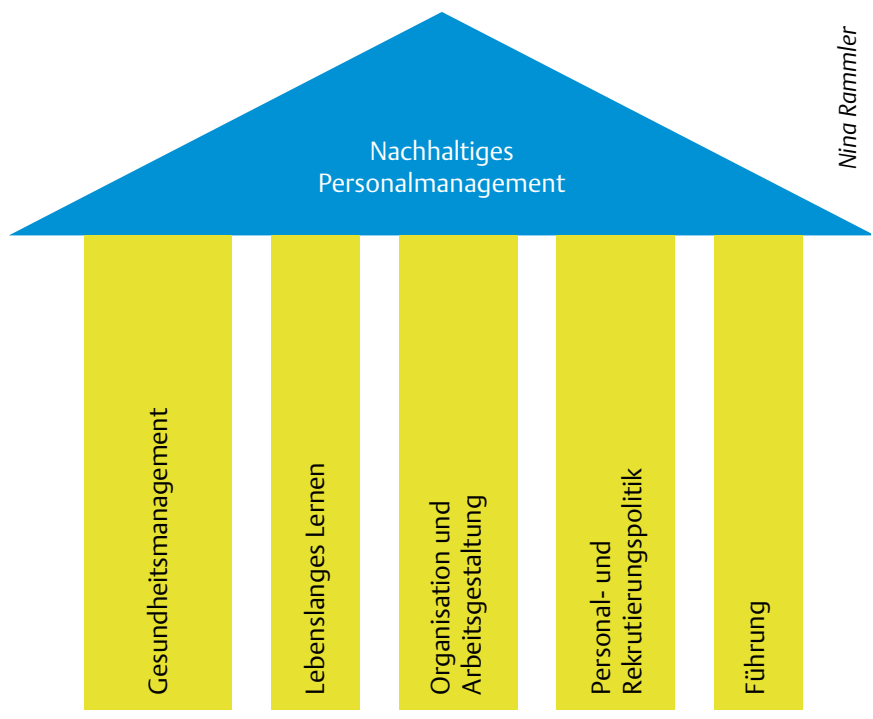

gesetzten besteht dort, wo Mitarbeiter gern zum Dienst kommen. Mitarbeiter, die sich auf ihre Vorgesetzten verlassen können und Weiterqualifizierung sowie Personalentwicklung auch tatsächlich erfahren, denen ermöglicht wird, ihre Expertise kontinuierlich auszubauen, arbeiten gern in diesem Unternehmen. Diese Mitarbeiter sind die besten Multiplikatoren für ein Unternehmen. Je mehr Multiplikatoren, also zufriedene Mitarbeiter ein Unternehmen hat, desto mehr wird sich dies auf das Employer Branding auswirken. Ein wirksames Employer Branding erreicht auch die für Kliniken wichtige jüngere Zielgruppe der zwischen 1980 und 1995 Geborenen. ${ }^{18}$

Schlechte Führungsqualität und Arbeitsunzufriedenheit oder besser gesagt ein schlechtes Employer Branding führen zu einer Abwanderung von Mitarbeitern, wenn die Erwartungen der Mitarbeiter nicht erfüllt werden. Die Führungsqualität steht in direktem Zusammenhang mit Mitarbeiterbindung und Arbeitszufriedenheit. ${ }^{9}$

Da sich die Arbeit von Pflegekräften grundlegend am Wohlergehen der Patienten orientiert, ist es für Unternehmen notwendig, pflegerischen Tätigkeiten große Aufmerksamkeit zu widmen, damit Pflegende die Möglichkeit haben, ihre beruflichen Wünsche (das Wohlergehen der Patienten) auch umzusetzen. Aber auch Kerngrößen wie Personalausstattung, Weiterqualifizierungsmöglichkeiten, Arbeitsaufteilung, Leistungsbewertung und Leistungszulagen sind direkt mit der Arbeitszufriedenheit der Mitarbeiter verbunden. ${ }^{9}$
Auf der Erwartungsliste ganz oben steht für Mitarbeiter die Dienstplansicherheit und -qualität. Spätestens beim Antritt der neuen Arbeitsstelle hat der Mitarbeiter Einblick in den Dienstplan. Es hat auch schon Bewerber gegeben, die sich beim Vorstellungsgespräch den Dienstplan von ihrem potenziellen neuen Arbeitgeber haben zeigen lassen. Aufgrund der verzeichneten Überstunden bei den Kollegen können Rückschlüsse auf Dienstplanqualität und Mitarbeiterwertschätzung gezogen werden. ${ }^{18}$

\section{Generationenorientiertes Human} Resources (HR) Management

Um langfristig den Bedarf an Pflegekräften sicherstellen zu können, bedarf es eines nachhaltigen Personalmanagements, das vielseitig und auf die Möglichkeiten und Ressourcen der Unternehmen angepasst sein soll. ${ }^{19}$

Das Fünf-Säulen-Modell bietet den Vorteil, dass die inhaltliche Ausgestaltung des Modells je nach Schwerpunktlage variabel ist. So können je nach Einrichtung und Ausprägung innerhalb der einzelnen Säulen Variationen stattfinden. Bei einer einrichtungsspezifischen Gestaltung, die an die entsprechende Situation angepasst ist, können die einzelnen Säulen unterschiedlich stark ausgeprägt sein ( $\rightarrow$ Abb. 3). Wichtig für ein nachhaltiges Personalmanagement ist jedoch, dass ein ausbalanciertes Konzept vorherrscht. Würden einzelne Säulen vollständig vernachlässigt und andere überdimensional stark ausgeprägt werden, wäre auch der Grundge- 

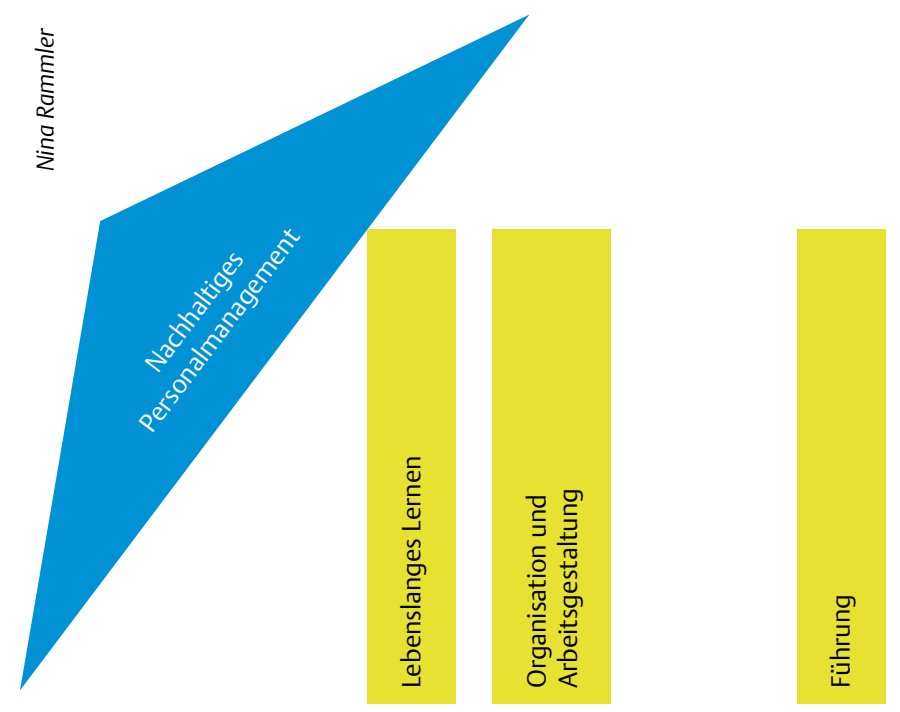

Abb. 4 Instabiles Modell des 5-SäulenKonzepts. ${ }^{19}$

danke der Nachhaltigkeit vernachlässigt. Als Folge davon würde das Modell instabil werden ( $\rightarrow$ Abb. 4). ${ }^{19}$

Gesundheitsmanagement. Beinhaltet nicht nur betriebliche Pflichtvorgaben der Gesundheitsfürsorge. Vielmehr ist Gesundheitsmanagement das Angebot seitens der Arbeitgeber, den Mitarbeitern Möglichkeiten zur sportlichen Betätigung zur Verfügung zu stellen. Auch präventive Gesundheitsmaßnahmen können ergriffen werden, wie das Angebot von Rückenschulprogrammen oder bestimmter Schutzimpfungen für die Mitarbeiter. Dies sollte altersgruppenübergreifend stattfinden. In betriebliches Gesundheitsmanagement zu investieren bringt auch wirtschaftliche Vorteile für das Unternehmen. So wird die Krankheitsquote durch die Förderung der Mitarbeitergesundheit gesenkt und es trägt zur Teamentwicklung bei. Das heißt, Gesundheitsmanagement umfasst sowohl präventive als auch kurative Instrumente. Angebote, die am Gesundheitsverhalten und Gesundheitsbewusstsein der Mitarbeiter ansetzen, beispielsweise Sportgruppen oder Ernährungsberatung, werden als Verhaltensprävention eingeordnet. Hingegen werden Optimierung der Arbeitsabläufe, Arbeitsplatzgestaltung und Führungsverhalten - z.B. Dienstplangestaltung - zur Verhältnisprävention gezählt. ${ }^{19}$

Angebote des Gesundheitsmanagements sollen bedürfnisorientiert sein und sowohl Bedürfnisse der Mitarbeiter, aber auch die des Unternehmens beinhalten.
Lebenslanges Lernen. Das menschliche Gehirn ist mit seinen Fähigkeiten, Erinnerungen zu speichern und wieder abzurufen, das vielleicht außergewöhnlichste Phänomen der Natur. ${ }^{20}$

Im Zusammenhang mit lebenslangem Lernen wird häufig der Begriff Personalentwicklung genannt. ${ }^{19}$ Sie fördert die Mitarbeiterbindung an den Betrieb, was sich wiederum auch wirtschaftlich auf das Unternehmen auswirkt. Dies wird auch als internes Personalmarketing bezeichnet. Ziel des externen Personalmarketings wäre es, im Rahmen des Employer Branding als attraktiver Arbeitgeber zu gelten und so neue, qualifizierte Mitarbeiter anzuwerben. ${ }^{19}$

Gleichzeitig ist lebenslanges Lernen die am häufigsten vernachlässigte Ressource des Personalmanagements. ${ }^{19}$ Der Wunsch nach einer beruflichen Weiterqualifizierung kristallisierte sich auch bei der NEXTStudie heraus. So dachten in Deutschland 28\% der Befragten mehrfach im Monat über eine Weiterqualifizierung nach. ${ }^{9}$ Die Möglichkeiten hierzu sind mittlerweile auch in Deutschland vielfältig. Wichtig für eine nachhaltige Personalentwicklung ist aufseiten des Unternehmens die Feststellung des Bedarfs an bestimmten Qualifikationen sowie die Einbeziehung der Mitarbeiter im Rahmen von Personalentwicklungsgesprächen.

„Die Attraktivität des Berufs kann nur gestärkt und Mitarbeiter im Berufsfeld Pflege können nur gehalten werden, wenn gut aus- und weitergebildete Pflegekräfte in ihrem Engagement belohnt werden und Unterstützung bei ihrer Karriereplanung erfahren." ${ }^{21}$

Organisation und Arbeitsgestaltung. Aufgrund arbeitsschutzrechtlicher Vorgaben und im Rahmen von physischen sowie psychischen Unterstützungsangeboten beispielweise das Angebot von Schulungen zu Mobilisations- und Lagerungstechniken - besteht ein sehr enger Bezug zum Gesundheitsmanagement, aber gleichzeitig auch zum Element des lebenslangen Lernens. Konkret geht es hier jedoch auch um das Belegungsmanagement einer Station, das an die aktuelle Personalsituation angepasst sein muss. Auch die Arbeitszeitregelung sowie die Dienstplangestaltung dürfen dabei nicht außer Acht gelassen werden. Grundvoraussetzung für die Organisation und Gestaltung des Arbeitsablaufs sind klare Arbeitsaufträge seitens der Führungskräfte. Dadurch wird eine Verbindung zum Element Führung hergestellt. Eine Überschneidung zum Element des lebenslangen Lernens wird beispielsweise durch das Ermöglichen von Rotationen der Mitarbeiter in verschiedenen Abteilungen des Hauses erreicht. Somit besteht für die Mitarbeiter die Option einer Weiterentwicklung, gleichzeitig können durch einen Perspektivenwechsel Prozesse und Arbeitsabläufe optimiert und verbessert werden. Obwohl es häufig ein Tabuthema ist und da aufgrund tarifrechtlicher Vorgaben bisweilen auch nur ein kleiner Spielraum besteht, ist an dieser Stelle das Thema Vergütung einzuordnen. Aber auch diesen Rahmen müssen Führungskräfte nutzen, um eine überdurchschnittliche Leistung oder ein besonderes Engagement einzelner Mitarbeiter zu honorieren. ${ }^{19}$

Personal- und Rekrutierungspolitik. Personalgewinnung und Mitarbeiterrekrutierung sind im Pflegebereich mittlerweile zu einem großen Wettbewerbsmarkt geworden. Krankenhäuser haben inzwischen Probleme, Fachkräfte für ihr Unternehmen zu gewinnen. Gleichzeitig steigt die Nachfrage nach qualifizierten Pflegekräften aufgrund der steigenden Zahlen an Pflegebedürftigen weiter an. Bereits 2007 hatte sich die Zahl der pflegebedürftigen Men- 

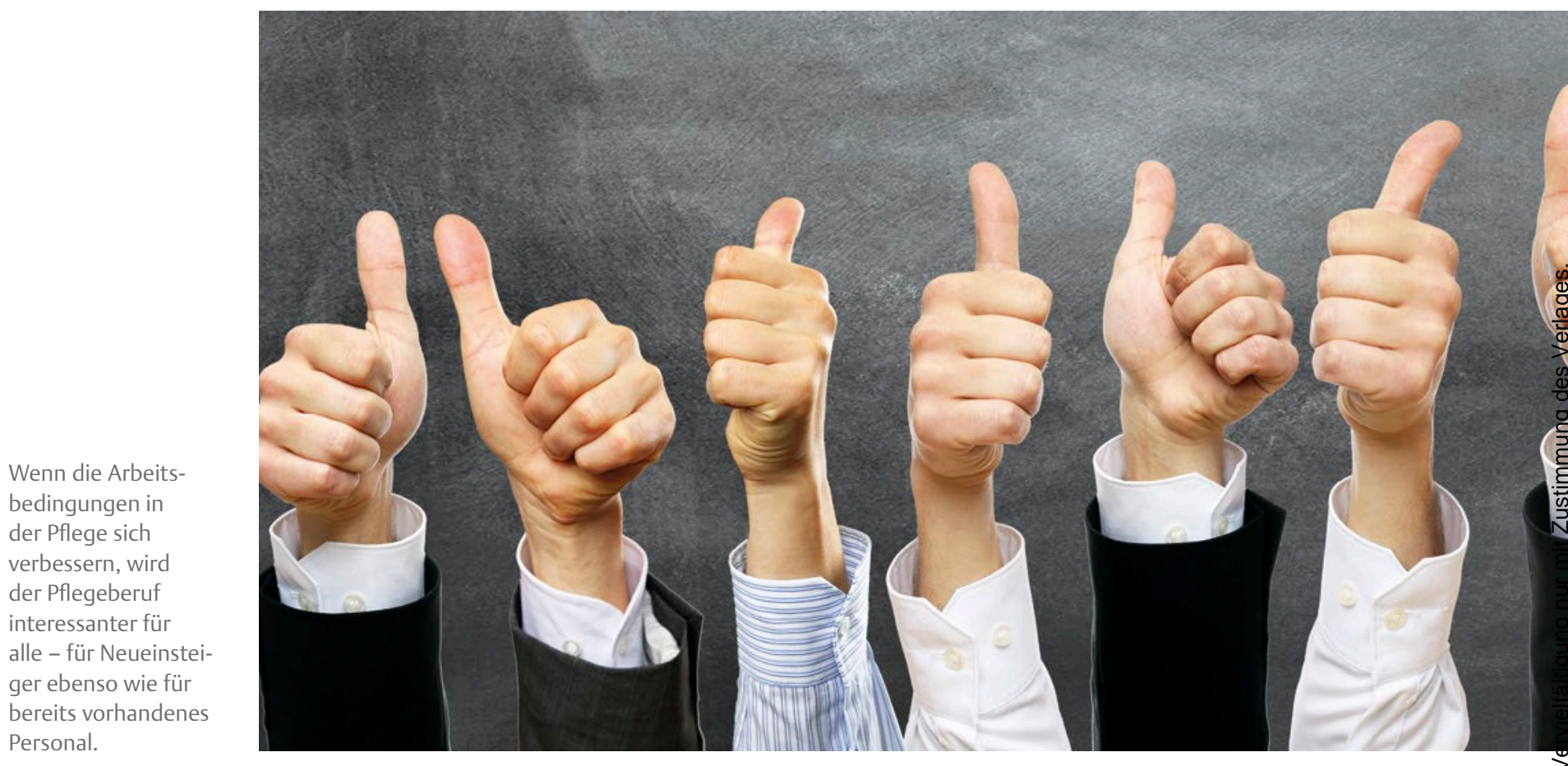

schen in Deutschland um 11,4\% gegenüber 1999 erhöht. ${ }^{22}$ Um dieser steigenden Zahl an Nachfrage für Pflegeleistungen in $\mathrm{Zu}-$ kunft gerecht zu werden, bedarf es einer Veränderung in der Personalpolitik der Unternehmen. Vor allem die Altersnachfolgeregelung würde sich durch ein nachhaltiges Personalmanagement vorausschauend planen lassen. ${ }^{19}$

Hierzu ist eine rechtzeitige Festlegung des Anforderungsprofils für zu besetzenden Stellen notwendig. Zudem wird eine Aufstellung der Anforderungskriterien für den weiteren Verlauf des Rekrutierungsmanagements essenziell. Es muss jedem Personalmanager klar sein, dass er unter Umständen eine gewisse Kooperationsbereitschaft gegenüber den Bewerbern zeigen muss. Denn ein Bewerber, der allen Anforderungen und Bedürfnissen gerecht wird und zusätzlich dem Unternehmen nur Vorteile bringt, ist auf dem Arbeitsmarkt nur schwer zu finden.

Neben den inhaltlichen Anforderungen einer Stellenausschreibung müssen auch die äußeren Anforderungen festgelegt werden. Dabei ist auf ein einheitliches Layout und eine den Leser ansprechende Gestaltung zu achten. Jede Stellenausschreibung, egal ob diese online oder in Printmedien erscheint, ist Öffentlichkeits- arbeit für das Unternehmen und gleichzeitig Imagepflege für die Berufsgruppe. ${ }^{19}$

Anzeigen und Plakatwände mit Slogans wie „Du weißt, auf welche Beats man nicht tanzen kann“ oder „Du weißt, womit man sich nicht die Haare schneidet“ und Ähnlichem mögen zunächst zwar witzig klingen. Wertschätzung gegenüber den Mitarbeitern und der Professionalität des Pflegeberufs wird jedoch kaum erbracht und mit generationenorientierter Personalgewinnung hat das nichts zu tun. ${ }^{23}$

Beim generationsgerechten Personalmarketing muss vielmehr darauf geachtet werden, dass die Stellenausschreibung den potenziellen Bewerber auch erreicht. Ergänzend zu Anzeigen in Printmedien muss dringend über die Option von Online-Jobbörsen nachgedacht werden. Ebenso können Instrumente wie Social Media Netzwerke vor allem junge Bewerber auf das Unternehmen und die Stellenangebote aufmerksam machen. ${ }^{19,23}$

Führung und die Rolle der Führungskraft. Unternehmen müssen in ihre Führungskräfte investieren, und diese wiederum müssen ihren Mitarbeitern Gehör schenken. Führungskräfte von heute müssen Vertrauen zu ihren Mitarbeitern aufbauen. Ehrlichkeit und Empathie gehören ebenso zum Eigenschaftskatalog einer Führungskraft wie Motivation der Mitarbeiter durch Unterstützung und Bestätigung in ihrem Tun und Handeln. ${ }^{19,24}$

Dreh- und Angelpunkt einer guten Personalpolitik wird immer die Führungskraft sein, denn durch sie werden alle anderen Elemente des Fünf-Säulen-Konzepts beeinflusst. Die Investition eines Unternehmens in gut ausgebildete und motivierte Führungskräfte ist lohnenswert, denn dies hat direkte Auswirkungen auf die Arbeitszufriedenheit der einzelnen Mitarbeiter. Vertrauen zu den Vorgesetzten ist die wichtigste Grundlage für eine gute Zusammenarbeit von Führungskräften und Mitarbeitern und bietet damit die Chance auf eine langfristige Mitarbeiterbindung an das Unternehmen. ${ }^{7}$

\section{Wer pflegt uns 2050?}

Eine explizite Lösung, um dem Pflegenotstand in Zukunft entgegenzuwirken, gibt es bisher nicht. Es können lediglich mögliche Lösungsansätze und Denkanstöße für ein zukünftiges Personalmanagement herausgearbeitet werden.

In der NEXT-Studie werden vier theoretische Möglichkeiten benannt, um die Anzahl der aktiven beruflich Pflegenden zu erhöhen. 
tung auf Augenhöhe mit anderen medizinischen Berufsgruppen auch auf Bundesebene zu ermöglichen. Es ist logisch und nachvollziehbar, dass sich vor allem die Politik sowie Arbeitgeberverbände gegen eine Interessenvertretung der Pflegenden in Form einer Pflegekammer aussprechen. Die Verweigerung einer Pflegekammer gegenüber den Pflegenden durch die bayerische Staatsregierung mit der Begründung, eine Pflegekammer sei den Pflegenden derzeit nicht zu vermitteln, kommt einem Kniefall vor den Arbeitgeberverbänden und Gewerkschaften gleich und bescheinigt der Staatsregierung nichts anderes als Konzeptlosigkeit und fehlende Akzeptanz des Pflegeberufs. Eine Befragung von beruflich Pflegenden der Hochschule München im Auftrag des Bayerischen Staatsministeriums für Umwelt und Gesundheit 2013 zeigt jedoch, dass sich zum Zeitpunkt der Befragung lediglich 34\% beruflich Pflegender gegen die Einführung einer Pflegekammer in Bayern ausgesprochen haben. ${ }^{28}$

In welcher Form auch immer - die Pflege braucht eine starke Selbstorganisation, um sich geschlossen vertreten zu können. Die Pflege muss erwachsen werden und lernen, für sich und ihre Interessen einzustehen. Solange sich Pflegende nur um andere kümmern, aber nicht um sich selbst, wird das Image des Pflegeberufs in der Bevölkerung, aber auch die Attraktivität des Berufs nicht zunehmen.

\section{Literatur}

1 Sermeus W, Aiken LH, Van den Heede K et al. Nurse forecasting in Europe (RN4CAST): Rationale, design and methodology. BMC Nursing 2011; 10: 6-14

2 Elmer C, Schäfer M. Wie die Pyramide zum Weihnachtsbaum wird. In. Spiegel Online I Wissenschaft: Der Spiegel; 2015

3 Müller R, Unger R, Rothgang H. „Pflege 2030“. Gefahr der Versorgungslücke. ZES Report 2013; 18. J.: 16-20

4 Isfort M, Weidner F, Neuhaus A et al. Pflege-Thermometer 2009. Eine bundesweite Befragung von Pflegekräften zur Situation der Pflege und Patientenversorgung im Krankenhaus. Köln: Deutsches Institut für angewandte Pflegeforschung e. V. (dip); 2010

5 Bundesagentur für Arbeit. Der Arbeitsmarkt in Deutschland. Fachkräfteengpassanalyse Dezember 2014. Nürnberg: Bundesagentur für Arbeit; 2014

6 Bundesagentur für Arbeit. Blickpunkt Arbeitsmarkt - Fachkräfteengpassanalyse. Dezember 2016. Nürnberg: Bundesagentur für Arbeit, Statistik/Arbeitsmarktberichterstattung; 2016
7 Fajardo A. Die (Un-) Attraktivität des Pflegeberufes in Deutschland und der Einfluss der Führungskräfte. Hamburg: Diplomica; 2013

8 Bomball J, Schwanke A, Stöver M, Schmitt S, Görres $S$. „Imagekampagne für Pflegeberufe auf der Grundlage empirisch gesicherter Daten“. Einstellungen von Schüler/innen zur möglichen Ergreifung eines Pflegeberufes. In: Institut für Public Health und Pflegeforschung der Universität Bremen ed. Bremen; 2010

9 Hasselhorn HM, Müller BH, Tackenberg P, Kümmerling A, Simon M. Berufsausstieg bei Pflegepersonal. Arbeitsbedingungen und beabsichtigter Berufsausstieg bei Pflegepersonal in Deutschland und Europa. Bremerhaven: Wirtschaftsverlag NW; 2005

10 Büker C. Arbeitsgestaltung in der Pflege. Arbeitsverteilung und Personaleinsatz. Studienbrief 3 1. ed. Hamburg: Hamburger Fern-Hochschule (HFH); 2012

11 Schambortski H. Mitarbeitergesundheit und Arbeitsschutz. Gesundheitsförderung als Führungsaufgabe. 1. ed. München: Elsevier, Urban \& Fischer; 2008: IX

12 Schwencke S. Pflege mit breitem Kreuz. CNEmagazin 2014: 16-18

13 Bundesagentur für Arbeit, Statistik. Arbeitsmarkt nach Berufen. Nürnberg; 2000-2017

14 Hornung J. Vom Personalmarketing zum Aufbau einer Arbeitgebermarke - nachhaltige Wege aus dem Personalmangel. In: Bettig U, Frommelt M, Schmidt R (Hrsg.). Fachkräftemangel in der Pflege - Konzepte, Strategien, Lösungen. Heidelberg: medhochzwei; 2012: 307-316

15 Schönberg G. Personalentwicklung als Chance für das Krankenhaus. In: Hellmann W, Hoefert HW (Hrsg.). Das Krankenhaus im demografischen Wandel - Theoretische und praktische Grundlagen zur Zukunftssicherung. Heidelberg: medhochzwei; 2012: 167-184

16 Weber-Ploemacher A, Röhm K, Döbler AK. Ärzte und Pfleger dringend gesucht. In. Düsseldorf: StepStone Deutschland $\mathrm{GmbH}$, Georg Thieme Verlag; 2012

17 Barwasser FM. Erwin Pelzig. Was wär' ich ohne mich? 2. ed. München, Zürich: Piper; 2003

18 Quernheim G. Als Arbeitgeber überzeugen. Die Schwester | Der Pfleger 2015; 54. Jg.: 74-77

19 Hornung J. Nachhaltiges Personalmanagement in der Pflege. Das 5-Säulen Konzept. Berlin, Heidelberg: Springer; 2013

20 Thompson RF. Das Gehirn. Von der Nervenzelle zur Verhaltenssteuerung. 3. ed. Heidelberg: Spektrum, Akademischer Verlag; 2001

21 Hoyer J, Borchert K. So finden Sie Ihre Schiene. intensiv 2014; 22: 322-326

22 Statistisches Bundesamt. Demografischer Wandel in Deutschland. Auswirkungen auf Krankenhausbehandlungen und Pflegebedürftigkeit im Bund und in den Ländern. Wiesbaden: Statistisches Bundesamt; 2010

23 Roedenbeck Schäfer M. Personalgewinnung in der Pflege. Innovative Ideen - einfach umgesetzt. Bewerberorientiert mit Social Media \& Co. 1. ed. München: Elsevier, Urban \& Fischer; 2014

24 Smerdka-Arhelger I. Auswirkungen der Personalpolitik auf die Organisationskultur und die Motivation der Mitarbeiter. In: Bechtel P, Friedrich D, Kerres A (Hrsg.). Mitarbeitermotivation ist lernbar - Mitarbeiter in Gesundheitseinrichtungen motivieren, führen, coachen. Berlin, Heidelberg: Springer; 2010: 223-237

25 Bundesanzeiger. Gesetzesbeschluss des Deutschen Bundestages. Gesetz zur Reform der Pflegeberufe (Pflegeberufereformgesetz - PflBRefG).
Drucksache 511/17. Köln: Bundesanzeiger; 2017: $1-47$

26 Maier J. „Sprechen Sie mich an!“. CNEmagazin 2014: 10-11

27 Schulz M. Neuverteilung von Aufgaben aller Gesundheitsberufe. Heilberufe 2014; 66: 63

28 Büker C, Lademann J, Mayer T et al. Befragung der beruflich Pflegenden zur Einrichtung einer Pflegekammer in Bayern. Abschlussbericht. München: Bayerisches Staatsministerium für Umwelt und Gesundheit, Bayerisches Staatsministerium für Gesundheit und Pflege; 2013

\section{Weiterführende Literatur}

Friedrich A. Management von Personal. Personalentwicklung. Studienbrief 4. 2. ed. Hamburg: Hamburger Fern-Hochschule; 2014

Conzen C. Pflegemanagement heute. Ökonomie, Personal, Qualität: verantworten und organisieren. 1. ed. München: Elsevier Urban \& Fischer; 2009

\section{Autorin}

\section{Nina Rammler}

Fachgesundheits- und Krankenpflegerin für Anästhesie und Intensivpflege (DKG); Klinikum der LudwigMaximilians-Universität (LMU) München am Campus Großhadern, Klinik für Anästhesiologie, Interdisziplinäre Intensivstation ITS 3. Studiert seit juli 2013 berufsbegleitend, teilweise auch parallel zur Fachweiterbildung Pflegemanagement B.A. an der HamburgerFernhochschule (HFH) und schreibt in ihrer Bachelorarbeit derzeit über Personalausfallmanagementkonzepte zur Kompensation von kurzfristigen Personalausfällen.

E-Mail: nina.rammler@med.unimuenchen.de

\section{Co-Autorin der Facharbeit}

\section{Birte Spier}

Fachgesundheits- und Krankenpflegerin für Anästhesie und Intensivpflege; ISARklinikum GmbH, München, Intensivstation

\section{Bibliografie}

DOI 10.1055/s-0043-118878 Intensiv 2017; 25: 284-292

(c) Georg Thieme Verlag KG

Stuttgart · New York · ISSN 0942-6035 